\title{
Robert Castel, La montée des incertitudes
}

\section{Hélène Garner}

\section{(2) OpenEdition}

Journals

Édition électronique

URL : http://journals.openedition.org/travailemploi/1730

DOI : 10.4000/travailemploi.1730

ISSN : 1775-416X

Éditeur

DARES - Ministère du Travail

\section{Édition imprimée}

Date de publication : 30 septembre 2009

Pagination : 89-91

ISSN : 0224-4365

\section{Référence électronique}

Hélène Garner, «Robert Castel, La montée des incertitudes », Travail et Emploi [En ligne], 119 | juilletseptembre 2009, mis en ligne le 30 septembre 2011, consulté le 22 septembre 2020. URL : http:// journals.openedition.org/travailemploi/1730; DOI : https://doi.org/10.4000/travailemploi.1730 


\section{La montée des incertitudes} Robert Castel

\author{
Seuil, 2009
}

\section{Lu par Hélène Garner(1)}

S'inscrivant dans la poursuite de la réflexion engagée en 1995 dans Les métamorphoses de la question sociale, le dernier ouvrage de Robert Castel, La montée des incertitudes, est un recueil de textes parus entre 1995 et 2008 et retravaillés pour certains par l'auteur en vue de cette publication. L'avant-propos, intitulé «Une grande transformation» (en référence à l'ouvrage de Polanyi publié en 1944), est une contribution originale qui synthétise en une cinquantaine de pages la réflexion et l'analyse du sociologue sur la grande transformation qu'a connue le salariat depuis une trentaine d'années et esquisse des pistes d'action pour réduire cette montée des incertitudes.

La montée des incertitudes est un ouvrage engagé; l'auteur s'invite dans les débats actuels (sur les politiques de diversité, le RSA, la sécurité sociale minimum garantie...) et son analyse se veut délibérément inscrite dans la sphère politique.

L’ouvrage est découpé en trois parties; la première, intitulée «Les dérégulations du travail», est composée de textes qui traitent de l'évolution de la place du travail dans la société actuelle, du rôle du droit du travail dans le redéploiement de l’État social, du rapport au travail spécifique des jeunes et de l'institutionnalisation du "précariat», forme d'activité au deçà du salariat. Ces phénomènes participent de ce que Castel appelle la grande transformation du monde salarial qui se caractérise par deux phénomènes de fond: l'individualisation de la relation de travail et le démantèlement des régulations collectives conduisant à un accroissement de l'insécurité pour les individus.

Après avoir rappelé les grands fondements de la société salariale qui s'est construite au cours des deux premiers tiers du $\mathrm{XX}^{\mathrm{e}}$ siècle, Castel analyse le processus de «décollectivisation » qui marque les rapports de travail depuis une trentaine d'années. Ce processus repose sur deux changements profonds: un changement dans l'organisation du travail avec d'une part le développement de petites unités de production recourant aux contrats précaires et à la sous-traitance et le démantèlement des grands bastions industriels, et d'autre part l'individualisation des tâches qui exige adaptabilité et prise de responsabilités des salariés. Et un changement dans les trajectoires professionnelles des salariés caractérisées jusqu'alors par la stabilité et la sécurité et aujourd'hui marquées par des ruptures et des interruptions générant de l'insécurité.

(1) Dares, mission animation de la recherche.
L'individu est au centre de ces évolutions et reconfigurations car sa responsabilisation et son autonomie sont au cœur de ce processus de «décollectivisation». Associées depuis la révolution française et la Déclaration des droits de l'homme et du citoyen de 1789 à la propriété privée, la liberté et la responsabilité de l'individu passent également depuis l'avènement de la société salariale par la propriété de droits attachés à la condition de salarié. Cette propriété sociale protège l'individu qui n'est pas protégé par la propriété privée et lui confère le statut de citoyen social(2). Cet individu moderne, s'il reste majoritaire, a été ébranlé par les reconfigurations de la société salariale et deux profils d'individus "hypermodernes » ont émergé conduisant à une bipolarisation de la société: les individus les plus faibles, les moins bien dotés en termes de capitaux matériels et intellectuels pour s'adapter aux changements, ceux que Castel qualifie «d'individus par défaut» (individus sans emploi et travailleurs précaires), et les «individus par excès » qui disposent de toutes les ressources pour être pleinement libres et responsables. Castel fustige la vision individualiste et globalisante de la société promue par les politiques qui ne distingue pas les individus en fonction de leurs ressources. Il dénonce les discours culpabilisateurs à l'encontre des chômeurs accusés de "l'être de façon volontaire et de vivre aux crochets de la France qui se lève tôt», qui conduisent à accroître les clivages entre les «individus par défaut» et les «individus par excès ». Car s'il place le travail au centre de son analyse, au centre de ce qui fonde l'identité d'un individu, son statut dans la société et la protection dont il bénéficie, il estime que la célébration du travail ne doit pas être assortie d'une stigmatisation de tous ceux qui ne travaillent pas ou sont assistés, mais d'une réflexion sur la manière de rendre tous les individus autonomes et responsables.

Cet effritement du compromis salarial et le démantèlement des régulations et protections collectives qui lui étaient associées concourent donc à accroître l'incertitude dans laquelle évoluent les individus et cette incertitude est synonyme de risque. La référence au risque est omniprésente dans notre société, note Castel, tant dans les discours politiques que médiatiques ou scientifiques. Il met en exergue trois évolutions attestant de la place croissante du risque dans notre société:

- la nécessaire reconfiguration du risque social: le système d'assurance mis en place avec la sécurité sociale dans l'après-guerre est aujourd'hui fragilisé car confronté à deux défis : un défi relatif à son financement dans un contexte où une part croissante de la population active se retrouve à la marge du travail, or c'est sur ce dernier que repose la logique assurantielle. Et un défi consécutif à l'apparition de

(2) Castel rappelle que l'un des premiers actes de l'Assemblée législative de 1789 a été d'exclure du droit de vote tous ceux qui ne disposaient pas d'un minimum de propriété, soit un tiers de la population en âge de travailler. 
nouveaux risques sociaux à gérer comme le risque dépendance;

- l'élaboration de «populations à risques » dont le profil est défini en partie de manière décontextualisée, par le recoupement de données statistiques, et en l'absence parfois de toutes interactions sociales, par des experts forcément éloignés, dans la représentation un peu caricaturale de l'auteur, des réalités du terrain. Cette vision de la société découpée en «populations à risques » accentue notre sensibilité aux risques et favorise son instrumentalisation par le politique en mettant l'accent sur la prévention des risques et la mise en place de mesures coercitives avant même la survenue des faits. On comprend à la lecture de ce paragraphe la méfiance qu'inspire à Robert Castel l'usage fait actuellement des statistiques, qui sont par ailleurs globalement peu mobilisées dans l'analyse présentée;

-l'apparition depuis les années 1980 de nouveaux risques résultant, comme un effet boomerang, des progrès scientifiques et biologiques réalisés depuis l'après-guerre (risque nucléaire, toxique, lié aux pesticides...).

Castel estime que l'éradication de tous les risques possibles, le mythe d'une société sans risque, est impossible et qu'il faut plutôt élaborer ce qu'il appelle une déontologie face aux risques, permettant d'y faire face au cas par cas, et déconstruire la conception globalisante du risque actuellement dominante.

La configuration de l'État social caractérisé par des régulations collectives et un rôle important dévolu aux syndicats et à la négociation, n’est donc plus en phase avec les évolutions du monde du travail et les besoins des individus et n'assure plus leur protection face aux risques multiples. On peut regretter la faible place accordée dans cette analyse à l’acteur syndical car ces processus de reconfiguration ont également fragilisé son statut, son rôle, ses missions traditionnelles et le contraignent, comme l'État, à repenser ses modalités d'action pour survivre à ces transformations.

Une fois ces constats établis, la deuxième partie de l'ouvrage s’intéresse aux reconfigurations nécessaires des protections individuelles et au rôle de l'État social dans un environnement mouvant et incertain, marqué par la mondialisation. Dans un contexte où les régulations collectives traditionnelles sont déstabilisées et ne parviennent plus à protéger tous les individus, il réaffirme la nécessité d'un État social fort et esquisse des pistes d'action dans ce sens.

Castel interroge la notion même de protection, analyse sa dimension socio-anthropologique et explique combien le type de protection existant dans une société relève d'un choix social et de choix politiques. Analysant successivement le réformisme de droite puis de gauche, il esquisse les voies d'un réformisme nécessaire, orienté vers la défense de l'intérêt général tout en s’adaptant aux lois du marché et aux nouvelles conditions d'emploi et de travail. Castel dessine deux voies de reconfigura- tion de l'État: au niveau de son périmètre d'action car même si l'État social s'est historiquement construit dans un cadre national, son action s'inscrit aujourd'hui dans une dimension mondiale et en l'absence d'institutions européennes et internationales susceptibles de rétablir la protection dont bénéficiaient les individus dans la société salariale, lui seul, estime Castel, peut encore réduire l'arbitraire des relations entre salarié et employeur. Et le sociologue fait du droit du travail un instrument privilégié de cette reconfiguration pour garantir la protection des travailleurs non assurée par les mécanismes de régulation traditionnelle, ceux qui se situent entre le salariat et l'indépendance, dans ce que Castel appelle les «zones grises » de l'emploi. S’inscrivant dans la poursuite des réflexions conduites par des économistes, des sociologues et des juristes sur la «flexicurité», il propose par exemple la mise en place d'une sécurité sociale minimale garantie, assise sur une dizaine de droits comme le droit à la santé, le droit au logement, le droit à des prestations décentes lorsque l'individu n'est plus en capacité de travailler, le droit à la formation... Cette sécurité minimale constituerait l'ossature de la citoyenneté sociale, indispensable selon Castel pour faire de tous les individus des citoyens de droit quel que soit leur statut d'emploi. Si l'auteur justifie et argumente la nécessité selon lui d'assurer cette citoyenneté sociale pour «sortir par le haut» des mutations décrites, il laisse en suspens les questions, pourtant centrales aujourd'hui, relatives à la façon d'organiser et de financer cette permanence des droits susceptible d'assurer aux travailleurs mobiles une plus grande sécurité.

Un second axe de redéploiement de l'État est de le doter de moyens lui permettant de mieux répondre aux besoins des individus en se plaçant au plus près d'eux, tout en les responsabilisant davantage en contrepartie. Mais le sociologue souligne les risques de cette individualisation de la protection et des ambiguïtés en relevant. Car, rappelle l'auteur, tous les individus n'ont pas les mêmes ressources, les mêmes capitaux pour entrer dans la logique actuelle de contrepartie, de droits et de devoirs, prônée par les pouvoirs publics: «[...] la capacité d'être un individu n'est pas donnée d'emblée et une fois pour toutes, parce que l'individu n'est pas une substance mais une construction historique» (p. 26). Les mesures d'activation des dépenses publiques et notamment sociales mises en œuvre par les pouvoirs publics renvoient pourtant à cette volonté de responsabiliser l'individu sans tenir compte de ses moyens pour le faire. Il illustre également ces risques par les effets pervers des processus de décentralisation censés assurer une réponse au plus près des besoins 
des individus et pouvant en réalité aboutir à des inégalités dans l’accès aux droits(3).

La dernière partie, Les chemins de la désaffiliation, est consacrée aux voies qui conduisent certains groupes à la fragilisation extrême, voire la rupture du lien social, ce que Castel a appelé dans Les métamorphoses de la question sociale, la désaffiliation. Ce qu'il y a de nouveau, souligne Castel, c'est que la désaffiliation menace des populations qui étaient encore récemment intégrées dans la société salariale et protégés par elle et qui, sans en être exclues, se retrouvent maintenant à la marge. La progression de l'exclusion tient selon lui aux évolutions décrites dans la première partie de l'ouvrage: aggravation de la déstabilisation de la condition salariale et individualisation des rapports de travail. Il centre son analyse sur quatre groupes désaffiliés ou en voie de désaffiliation et relie à chaque fois leur condition aux évolutions et reconfigurations sus-décrites: les marginaux, les exclus, la classe ouvrière, les minorités ethniques. Pour ce dernier groupe, estimant que l'espace urbain est devenu le réceptacle privilégié de la question sociale à travers la mise en œuvre des politiques de la ville notamment, Castel relie les deux questions, urbaine et sociale, et affirme la nécessité de penser la question urbaine et la problématique des quartiers à travers les transformations du monde salarial; car selon lui c'est d'abord l'absence de travail qui caractérise ces quartiers. Concernant la question ethnique, il estime qu'elle recouvre les deux dimensions, ethnique et sociale, ce qui rend leur association explosive car leurs effets s’additionnent. Mais pour Castel, c'est toujours la dimension sociale qui prime et la question ethnique ne fait que la complexifier davantage; c'est donc sur la dimension sociale qu'il convient d'abord de travailler, l'enjeu étant de permettre aux minorités ethniques d'accéder aux conditions de leur «indépendance sociale». Car pour le sociologue la citoyenneté politique n’est qu'illusoire sans la citoyenneté sociale. Et c’est le rôle de l’État, car lui seul est en mesure de le faire, de chercher à «réaffilier » ces populations et d'en faire des individus à part entière.
(3) Un rapport sur la gouvernance des politiques territoriales financé par la Dares présente une analyse de ces effets fondés sur des observations de terrain. «Émergence, enjeux et risques de la gouvernance territoriale des politiques sociales», Jean-Michel Herbillon, Agnès Gramain et Samuel Neuberg, Contribution française au projet de recherche européen Rescaling Social Welfare Policies: A Comparative Study on the Path Towards Multi-level Governance in Europe (2005-2008), Université de Nancy-II, Centre d'études de l'emploi, novembre 2008, 208 p. 